
can methodically compare different encapsulation systems side by side, rather than tinkering through trial and error with whatever leftover $\beta$-cells they could get their hands on - the "dregs of material", as Melton puts it. And this rational engineering approach is leading to improvements in the design of both large and small cell-safeguarding techniques.

Some companies, including the regenerativemedicine heavyweight ViaCyte, based in San Diego, California, are loading thousands of $\beta$-cells into macroencapsulation devices that are as big as the palm of your hand. Others, like the start-up Sigilon, based in Cambridge, Massachusetts, are parcelling up individual bundles of $\beta$-cells into microscopic shells smaller than a mustard seed. With both strategies, "we're at this inflection point" where success is within reach, says Julia Greenstein, vice-president of discovery research at JDRF in New York City, a non-profit formerly known as the Juvenile Diabetes Research Foundation. "We've seen a much more scientifically directed approach to the problem than ever before."

\section{STEALTH MODE}

For many years, the most popular capsule material for transplanted $\beta$-cells has been a seaweed extract called alginate. It's been tested in rodents, dogs, monkeys and even humans in a few pilot clinical studies. The human trials showed that the material was safe, even in people who were not taking immunosuppressant drugs. But the therapeutic benefit was marginal because, within weeks of implantation, the alginate would usually begin to attract immune cells such as macrophages and neutrophils. This led to the deposition of fibrotic scar tissue that gummed up the capsules, choking off the cells inside.

This type of immune reaction was different from the one that originally destroyed the patients' $\beta$-cells, but it was equally damaging to the prospects of this therapeutic approach. Seeking a derivative of alginate that could evade immune detection altogether, Anderson teamed up with his MIT colleague Robert Langer. The researchers systematically screened close to 800 chemical offshoots of alginate in mice. They found one variety triazole-thiomorpholine dioxide alginate that seemed to go completely unnoticed by the immune system ${ }^{2}$.

Tiny spheres of this super-alginate survived for up to six months when implanted in macaques. And, when loaded with Melton's stem-cell-derived $\beta$-cells, the capsules could restore typical levels of blood sugar in a mouse model of T1D, with no signs of an immune reaction". "We demonstrated, for the first time, a material that remains fibrosis-free when implanted in the body," says Omid Veiseh, a biomaterials researcher who worked on the project as a postdoc at MIT. “There hasn't been anything like this."

Veiseh will start his own lab in 2017 at Rice

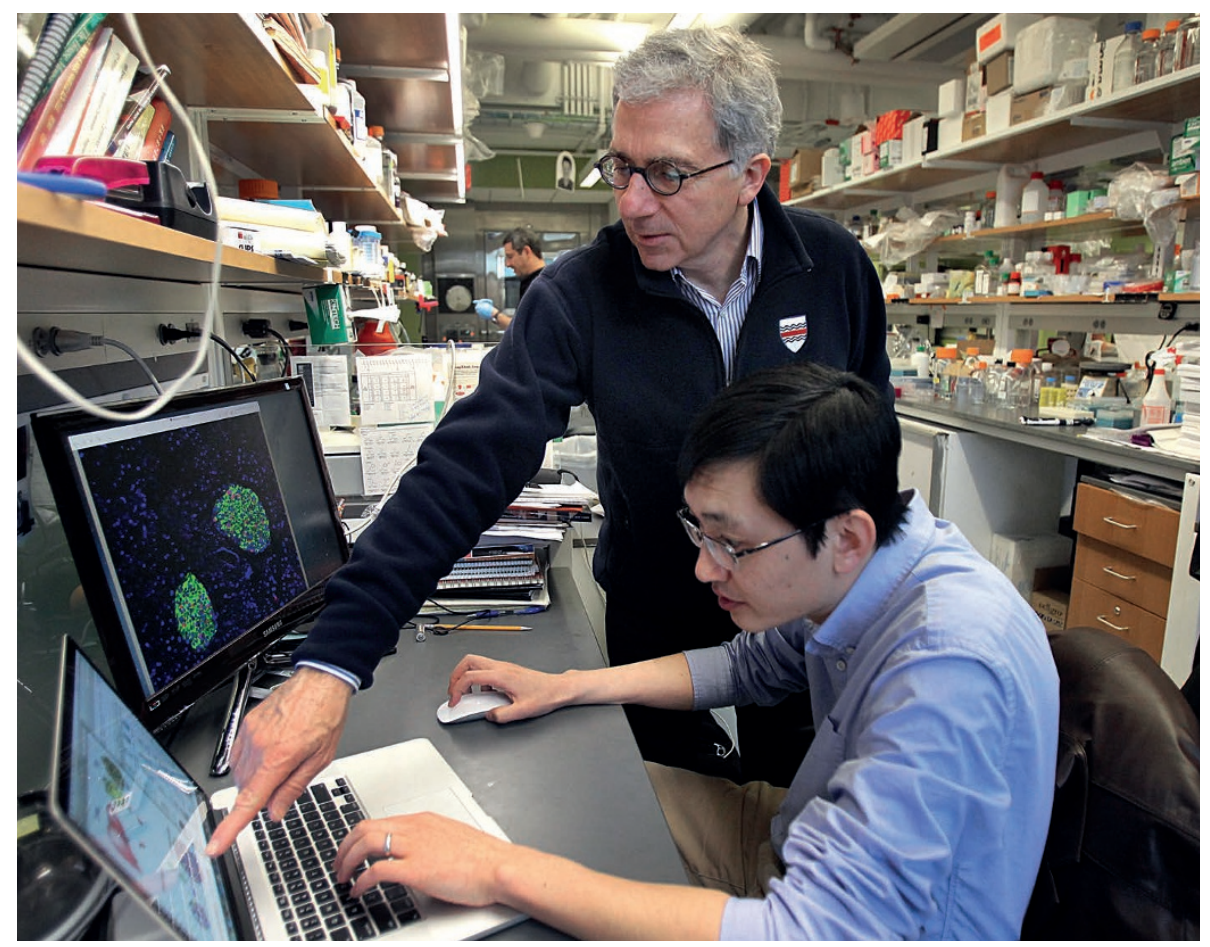

Douglas Melton (back), co-director of the Harvard Stem Cell Institute, has found a way to engineer $\beta$-cells.

University in Houston, Texas. But until then, he is sticking around in Cambridge to help get Sigilon off the ground. Named after the Spanish word for stealth, Sigilon launched this year with US\$37.5 million in funding to commercialize the new alginate for a variety of biomedical applications. These include two potential ways to treat T1D. One is microencapsulated cell therapy. By 2018, Sigilon intends to show that this technology works in people using donor islets, and it is looking to partner with cell-therapy companies about then testing a stem-cell-derived $\beta$-cell enveloped in its alginate. The second approach is to use the material to coat parts of bionic pancreas systems that enter the body (see 'Bionic versus beta'). "One way or another," says Sigilon president and chief executive Paul Wotton, patients with T1D will "benefit from this platform."

\section{SMALL TALK}

In the microencapsulation field, size matters. In tests with the super-alginate, the MIT team used capsules with a diameter of 1.5 millimetres, which it has demonstrated are much less immunogenic than the $0.5-\mathrm{mm}$ capsules that researchers in the diabetes cell-therapy field have conventionally used ${ }^{4}$. But a tripling of the diameter means a nearly 30 -fold increase in the volume of each capsule. And given the large number of capsules required to contain the hundreds of millions of $\beta$-cells needed to control a person's diabetes, there are few places in the body where the therapy could be implanted.

The capsules probably won't fit under the skin or in another easily retrievable location - and regulatory agencies have insisted, as a safety measure, that any stem-cell-derived diabetes therapy implanted in patients should be fully recoverable. That's why Alice Tomei, a bioengineer at the University of Miami in Florida, has developed what she calls a "shrink-wrapping technology", which uses microfluidics to apply a thin biocompatible coating to clusters of cells to make the smallest possible capsule - one that's only about $0.2 \mathrm{~mm}$ across ${ }^{5}$. Her material of choice, polyethylene glycol, may be more immune-reactive than Sigilon's super-alginate, but Tomei argues that her thinly wrapped cells

"The dream
is to be able
to build
some kind
of immuno-
isolation
device."
will be small enough to implant in more accessible spots in the body.

Tomei is evaluating her technology using Melton's stemcell-derived $\beta$-cells, in collaboration with start-up Semma Therapeutics in Cambridge, Massachusetts. Melton launched Semma in 2015 with the biotech investor and entrepreneur Robert Millman. (Millman's wife came up with the name: a combination of Sam and Emma, the names of Melton's two diabetic children.) Although Semma has its own encapsulation technology through the acquisition of drug-delivery-technology company Cytosolv, it is also looking for partners such as Tomei to test a range of encapsulation systems with the company's stem cells. "Anyone who's got a device, we'll work with them," says Millman, Semma's chief executive, "because even with the best cells, if we don't have the right device it'll fail."

Another of Semma's collaborators is Beta- $\mathrm{O}_{2}$ Technologies, a company based in 


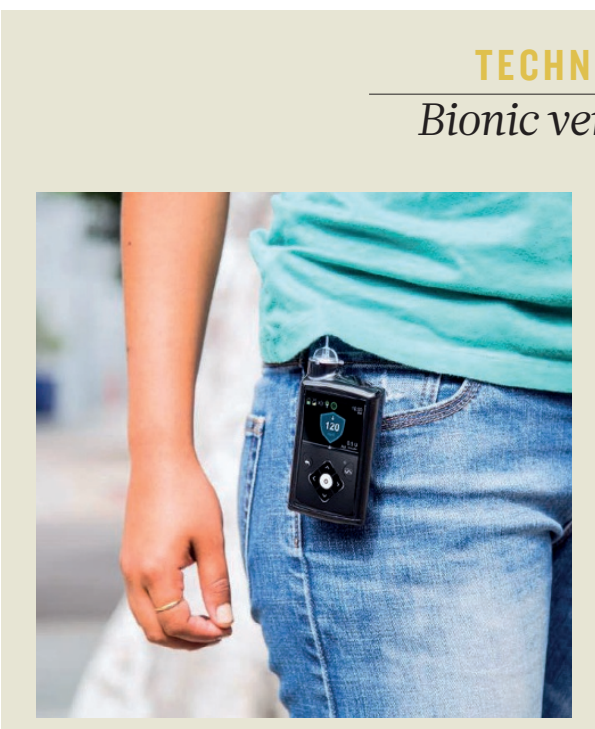

In September 2016, the US Food and Drug Administration approved the first medical device that can both supply insulin when blood sugars are high and shut off insulin delivery when sugar levels start to drop, with little input from the patient. This artificial pancreas system — the MiniMed 670G from Medtronic, a medical device company headquartered in Dublin combines two devices, one that monitors blood glucose levels and one that infuses insulin, with an algorithm that automates the delivery of insulin.

The bulk of the MiniMed set-up, including most of the electronics and the insulin pump, sits outside the body in a mobile-phone-sized device (pictured) that people can carry in a pocket or wear on a belt. This device is attached to a tiny tube that is inserted beneath the skin. Much of the glucose-monitoring system is found in a patch worn on the surface of the abdomen, but this is also connected to a subcutaneous sensor.

The components that go into the body are prone to fibrotic immune reactions, and thus currently need to be replaced every few days. Coating the glucose sensor or the insulin-delivering catheter with a less immune-reactive material, such as the one being developed by Sigilon, a start-up in Cambridge, Massachusetts, could make the components last longer. But patients still need to tell the MiniMed (and similar technologies) when they've exercised or eaten - and no device can offer bloodglucose control that's anywhere near as tight as that provided by the body's own cells.

That's why Douglas Melton, co-director of the Harvard Stem Cell Institute in Cambridge, Massachusetts, who has two children with type 1 diabetes, continues to work on a cell-replacement therapy for the disease that would add back the insulin-producing $\beta$-cells that are lost. His benchmark for success: "It has got to make my children have a life in which they don't think any more about what they're going to eat or how much they've exercised any more than you and I do." E.D.

once a week. But the company needs a more reliable cell source, and Avni has high hopes for Melton's cells.

\section{BOUNDING INTO THE CLINIC}

Melton's original recipe for making $\beta$-cells was cumbersome. It took 35 days of carefully swapping 5 different growth media and mixing in 11 different factors, including sugars and proteins. According to Felicia Pagliuca, a former postdoc in Melton's lab who now leads cellbiology research and development at Semma, she and her team have dramatically streamlined the protocol. "We are leaps and bounds further from where we were," she says. And they have a strategy for getting the cells into clinical trials, even before an encapsulation device is ready.

The plan is to make $\beta$-cells from induced pluripotent stem cells created from people who need insulin, but whose bodies don't attack their own cells, which happens in people with T1D. Since there would be no tissue mismatch or chance of autoimmune reaction, those cells could then be implanted back into the patients without any immune-suppressing drugs or barrier technologies.

Semma is focused on three patient populations, none of which have autoimmunity: people with a form of type 2 diabetes called lean diabetes, in which $\beta$-cells have stopped working; individuals who have had their pancreases surgically removed because of problems such as chronic inflammation; and patients with an insulin-dependent form of cystic fibrosis. The company hopes to test its cells in one of these populations in three to four years; trials with any sort of encapsulated device for people with T1D will follow at a later point.

But this means that Semma might be playing catch-up with its competitor ViaCyte. Earlier this year, the firm absorbed one of its chief rivals, a division of Johnson \& Johnson called BetaLogics, while also announcing promising early data from the first human trial of an encapsulated stem-cell-derived product for T1D.

ViaCyte's PEC-EnCap device is made up of a semi-permeable pouch, about the size of a sticking plaster, that contains thousands of pancreatic precursor cells, each derived from embryonic stem cells. The company uses precursor cells, rather than fully mature $\beta$-cells, because these cells are hardier under the lowoxygen conditions that follow implantation, when the packets haven't yet integrated with the blood system. Over the past 2 years, ViaCyte has implanted its devices under the skin of about 20 patients without immunosuppression. In many recipients, the pancreatic precursors have grown into insulin-producing $\beta$-cells - although these cells often die after a few months, owing to a fibrotic immune reaction on the device exterior.

"That's a proof of feasibility that this is achievable, but we still have a lot of work to do," says ViaCyte's chief executive, Paul Laikind. "The goal now is to reduce or delay that foreign-body response long enough for the cells to engraft." ViaCyte hopes to achieve this by modifying either the encapsulation device or some other aspect of the treatment protocol before it moves into the next phase of testing with a full therapeutic doses of its product.

By then, perhaps, Melton's $\beta$-cells could also be ready for testing in patients with T1D. Melton is confident that with the right delivery system, these cells can cure his children's illness. "It just makes sense to me," Melton says, "that if you can make the cell that's missing in a person we ought to be able to find a way to put that cell back into people."

Elie Dolgin is a science writer in Somerville, Massachusetts.

1. Pagliuca, F. W. et al. Cell 159, 428-439 (2014).

2. Vegas, A. J. et al. Nature Biotechnol. 34, 345-352 (2016).

3. Vegas, A. J. et al. Nature Med. 22, 306-311 (2016).

4. Veiseh, O. et al. Nature Mater. 14, 643-651 (2015).

5. Tomei, A. A. et al. Proc. Natl Acad. Sci. USA 111, 10514-10519 (2014)

6. Ludwig, B. et al. Proc. Natl. Acad. Sci. USA 110, 19054-19058 (2013) 